\title{
STABLE AND UNIFORMLY STABLE UNIT BALLS IN BANACH SPACES
}

\author{
ANTONIO SUAREZ GRANERO
}

\begin{abstract}
Let $X$ be a Banach space with closed unit ball $B_{X}$ and, for $x \in X$, $r \geq 0$, put $B(x ; r)=\{u \in X:\|u-x\| \leq r\}$ and $V(x, r)=B_{X} \cap B(x ; r)$. We say that $B_{X}$ (or in general a convex set) is stable if the midpoint map $\Phi_{1 / 2}: B_{X} \times B_{X} \rightarrow B_{X}$, with $\Phi_{1 / 2}(u, v)=\frac{1}{2}(u+v)$, is open. We say that $B_{X}$ is uniformly stable (US) if there is a map $\alpha:(0,2] \rightarrow(0,2]$, called a modulus of uniform stability, such that, for each $x, y \in B_{X}$ and $r \in$ $(0,2], V\left(\frac{1}{2}(x+y) ; \alpha(r)\right) \subseteq \frac{1}{2}(V(x ; r)+V(y ; r))$. Among other things, we see: (i) if $\operatorname{dim} X \geq 3$, then $X$ admits an equivalent norm such that $B_{X}$ is not stable; (ii) if $\operatorname{dim} X<\infty, B_{X}$ is stable iff $B_{X}$ is US; (iii) if $X$ is rotund, $X$ is uniformly rotund iff $B_{X}$ is US; (iv) if $X$ is 3.2.I.P, $B_{X}$ is US and $\alpha(r)=r / 2$ is a modulus of US; (v) $B_{X}$ is US iff $B_{X^{* *}}$ is US and $X, X^{* *}$ have (almost) the same modulus of US; (vi) $B_{X}$ is stable (resp. US) iff $B_{C(K, X)}$ is stable (resp. US) for each compact $K$ iff $B_{A(K, X)}$ is stable (resp. US) for each Choquet simplex $K$; (vii) $B_{X}$ is stable iff $B_{L_{p}(\mu, X)}$ is stable for each measure $\mu$ and $1 \leq p<\infty$.
\end{abstract}

\section{INTRODUCTION}

Let $X$ be a normed space and $B_{X}$ and $S_{X}$ the closed unit ball and unit sphere of $X$, respectively. If $A, B$ are subsets of $X$, define the distance $d(A, B)=\sup _{x \in A} \inf _{y \in B}\{\|x-y\|\}$. If $x \in X$ and $\varepsilon>0$, we write $B(x ; \varepsilon)=$ $\{y \in X:\|x-y\| \leq \varepsilon\}$ and $V(x ; \varepsilon)=B_{X} \cap B(x ; \varepsilon)$. We denote by $\operatorname{Ext}(C)$ the set of extreme points of a set $C$. A convex set $C$ is said to be stable if the midpoint map $\Phi_{1 / 2}: C \times C \rightarrow C, \Phi_{1 / 2}(u, v)=\frac{1}{2}(u+v)$, is open. Stable convex sets have been studied in $[4,12,3]$. Many Banach spaces have stable unit ball, namely: strictly convex or rotund Banach spaces, Banach spaces with 3.2.I.P. [4, p. 195], finite dimensional Musielak-Orlicz spaces [5], etc. In case of stable unit balls, the characterization of some extreme elements is very easy. For instance, if $K$ is a compact space, $B_{X}$ stable and $f \in C(K, X)$, then $f \in \operatorname{Ext}\left(B_{C(K, X)}\right)$ iff $f(K) \subseteq \operatorname{Ext}\left(B_{X}\right)$. If $K$ is a Choquet simplex, $B_{X}$ stable and $f \in A(K, X)$ (= affine continuous functions $g: K \rightarrow X$ ), then $f \in \operatorname{Ext}\left(B_{A(K, X)}\right)$ iff $f(\operatorname{Ext}(K)) \subseteq \operatorname{Ext}\left(B_{X}\right)$ (see [4, 2.1. Theorem]). Let $\mathscr{K}(X, C(K))$ be the space of compact operators $T: X \rightarrow C(K)$. It is known that $\mathscr{K}(X, C(K))$ is isometrically isomorphic to the space $C\left(K, X^{*}\right)$. Follow-

Received by the editors April 3, 1989 and, in revised form, January 15, 1990.

1980 Mathematics Subject Classification (1985 Revision). Primary 46B20.

Key words and phrases. Stable and uniformly stable sets, unit ball, Banach spaces.

Supported in part by DGICYT grant PB88-0141. 
ing Morris and Phelps [11], an operator $T \in \mathscr{K}(X, C(K))$ is said to be a nice operator, if its adjoint $T^{*}$ satisfies $T^{*}(K) \subseteq \operatorname{Ext}\left(B_{X^{*}}\right)$. Let $\mathscr{N} \mathscr{K}(X, C(K))$ be the set of nice operators. Then clearly $\mathscr{N} \mathscr{K}(X, C(K)) \subseteq \operatorname{Ext}\left(B_{\mathscr{K}}(X, C(K))\right)$ and, if $B_{X^{*}}$ is stable, we have $\mathscr{N} \mathscr{K}(X, C(K))=\operatorname{Ext}\left(B_{\mathscr{K}}(X, C(K))\right.$ (see $[2,15$, 16]).

If $x, y \in B_{X}$ and $s \geq 0$, denote $D(x, y ; s)=\frac{1}{2}[V(x ; s)+V(y ; s)]$. Obviously, $D(x, y ; s)$ is a convex and, in general, not norm closed set; but if $X$ is a dual space, then $D(x, y ; s)$ is $w^{*}$-compact and so norm closed. A map $\alpha:[0,2] \rightarrow[0,2]$ is a modulus of stability of $B_{X}$ if $V\left(\frac{1}{2}(x+y) ; \alpha(r)\right) \subseteq$ $D(x, y ; r)$ for each $x, y \in B_{X}$ and $r \in[0,2]$. The maximum modulus of stability $\alpha_{m}$ of $B_{X}$ is

$$
\begin{aligned}
r \in[0,2], \quad \alpha_{m}(r)=\sup \{s \in[0,2]: & \text { for each } x, y \in B_{X}, \\
& \left.V\left(\frac{1}{2}(x+y) ; s\right) \subseteq D(x, y ; r)\right\} .
\end{aligned}
$$

Clearly $\alpha_{m}$ is nondecreasing, null in $\left[0, r_{0}\right)$ and positive in $\left(r_{0}, 2\right]$, for some $r_{0} \geq 0$, and $\alpha_{m}(2)=2$. The closed unit ball $B_{X}$ is said to be uniformly stable (US) if there exists a map $\alpha:(0,2] \rightarrow(0,2]$ (called a modulus of US) such that for each $x, y \in B_{X}$ and $r \in(0,2]$ we have: $V\left(\frac{1}{2}(x+y) ; \alpha(r)\right) \subseteq D(x, y ; r)$. Of course, $B_{X}$ is US iff $\alpha_{m}(r)>0$ for each $r \in(0,2]$.

In this paper we study stable and uniformly stable (US) unit balls in Banach spaces. In $\S \S 1$ and 2 we see some elementary facts as: (i) if $\operatorname{Dim} X \geq 3, X$ admits an equivalent norm such that $B_{X}$ is not stable; (ii) if $X$ is a dual space, $\alpha_{m}$ is right continuous; (iii) if $\operatorname{Dim} X<\infty, B_{X}$ is stable iff $B_{X}$ is US; (iv) if $X$ is rotund, $X$ is uniformly rotund iff $B_{X}$ is US; (v) $B_{X}$ is US if $X$ is 3.2.I.P. In $\S 3$ we prove that $B_{X}$ is US iff $B_{X^{* *}}$ is US and the moduli of US are (almost) the same. $\S \S 4$ and 5 are devoted to study the unit balls $B_{C(K, X)}$ and $B_{A(K, X)}$, where $C(K, X)$ (resp. $A(K, X)$ ) is the space of continuous (resp. affine continuous) functions $f: K \rightarrow X$ on the compact $K$ (resp. convex compact $K)$. It is proved that $B_{X}$ is stable (resp. US) iff $B_{C(K, X)}$ is stable (resp. US) for each compact $K$ iff $B_{A(K, X)}$ is stable (resp. US) for each Choquet simplex $K$. In $\S 6$ we prove that $B_{X}$ is stable iff $B_{L_{p}(\mu, X)}$ is stable, $1 \leq p<\infty$. Finally, it is an open problem if $B_{L_{p}(\mu, X)}, 1 \leq p<\infty$, is US when $B_{X}$ is US.

\section{Preliminary Results}

We begin with some elementary remarks: (a) Let $C$ be a convex subset of some locally convex space $E, 0<\lambda<1$, and define $\Phi_{\lambda}: C \times C \rightarrow C$ by $\Phi_{\lambda}(x, y)=\lambda x+(1-\lambda) y$. Then $\Phi_{1 / 2}$ is open iff $\Phi_{\lambda}$ is open (see [4, 1.1 Proposition]).

(b) It is easily seen that $B_{X}$ is stable, if $X$ is a normed space with strictly convex or rotund norm.

(c) Every norm in $\mathbf{R}$ and $\mathbf{R}^{2}$ produces stable closed unit balls. In $\mathbf{R}$ this result is evident and, concerning $\mathbf{R}^{2}$, we can apply that, for a compact convex set $K \subseteq \mathbf{R}^{3}$, the map $\Phi_{1 / 2}$ is open iff $\operatorname{Ext}(K)$ is closed (see [4]). But if $z$ belongs to $S_{\mathbf{R}^{2}} \backslash \operatorname{Ext}\left(B_{\mathbf{R}^{2}}\right)$, there exist $x, y \in S_{\mathbf{R}^{2}} \backslash\{z\}$ such that $z=\frac{1}{2}(x+y)$. Let $\varepsilon=\|z-x\|$. Then $B(z ; \varepsilon / 2) \cap S_{\mathbf{R}^{2}} \subseteq S_{\mathbf{R}^{2}} \backslash \operatorname{Ext}\left(B_{\mathbf{R}^{2}}\right)$. Therefore $S_{\mathbf{R}^{2}} \backslash \operatorname{Ext}\left(B_{\mathbf{R}^{2}}\right)$ is open in $S_{\mathbf{R}^{2}}$ and hence $\operatorname{Ext}\left(B_{\mathbf{R}^{2}}\right)$ is closed.

(d) In $\mathbf{R}^{3}$ it is easy to give a norm such that $B_{X}$ is not stable. Take

$$
C=\left\{(x, y, 0): x^{2}+y^{2}=1\right\}, \quad K=\{(0, y, z): \max \{|y|,|z|\} \leq 1\} \text {. }
$$


The new closed unit ball will be $B=\operatorname{co}(C \cup K)$, that is, the convex hull of the set $C \cup K$. Observe that

$$
\operatorname{Ext}(B)=\{C \backslash\{(0,1,0),(0,-1,0)\}\} \cup\{(0, \pm 1, \pm 1)\} .
$$

As this set is not closed, by the result of [4] aforementioned in (c), $B$ is not stable.

If $\operatorname{dim} X \geq 3$, the situation is similar. We need the following lemma:

1.1. Lemma. Let $X$ be a normed space. Then: (A) If $B_{X}$ is stable, $\operatorname{Ext}\left(B_{X}\right)$ is closed.

(B) Let $Y \subseteq X$ be a 1-complemented subspace. If $B_{X}$ is stable, then $B_{Y}$ is stable.

Proof. (A) Let $\Delta=\left\{(x, x): x \in B_{X}\right\}$. As $\Delta$ is closed in $B_{X} \times B_{X}, \Phi_{1 / 2}$ open and $B_{X} \backslash \operatorname{Ext}\left(B_{X}\right)=\Phi_{1 / 2}\left(B_{X} \times B_{X} \backslash \Delta\right)$, then $B_{X} \backslash \operatorname{Ext}\left(B_{X}\right)$ is open and $\operatorname{Ext}\left(B_{X}\right)$ is closed in $B_{X}$.

(B) We say that $Y$ is 1-complemented in $X$ if there exists a projection $P: X \rightarrow Y$ such that $\|P\|=1$. Let $\Phi_{1 / 2}^{X}, \Phi_{1 / 2}^{Y}$ be the midpoint maps in $B_{X}$ and $B_{Y}$ respectively. Since $P \circ \Phi_{1 / 2}^{X}=\Phi_{1 / 2}^{Y} \circ(P \times P)$ and $P$ is open, we get that $\Phi_{1 / 2}^{Y}$ is open if $\Phi_{1 / 2}^{X}$ is open.

1.2. Proposition. Let $X$ be a normed space with $\operatorname{dim} X \geq 3$. Then $X$ admits an equivalent norm such that the new closed unit ball is not stable.

Proof. We write $X$ as a direct topological sum $X=X_{1} \oplus X_{2}$ with $\operatorname{dim} X_{1}=3$. Take in $X_{1}$ the norm $\|\cdot\|_{1}$ used in (d) above, an arbitrary equivalent norm $\|\cdot\|_{2}$ in $X_{2}$ and in $X$ the norm $\|\cdot\|_{3}$ defined by

$$
\text { if } x=\left(x_{1}, x_{2}\right) \in X_{1} \oplus X_{2}=X \text {, then }\|x\|_{3}=\sup \left\{\left\|x_{1}\right\|_{1},\left\|x_{2}\right\|_{2}\right\} \text {, }
$$

Now it is enough to consider (d) and Lemma 1.1.

1.3. Proposition. Let $X=Y^{*}$ be a dual Banach space. Then $\alpha_{m}$ is rightcontinuous.

Proof. Let $s_{0} \in[0,2), \varepsilon>0$ and suppose that $\lim _{s \rightarrow s_{0}^{+}} \alpha_{m}(s)=\alpha_{m}\left(s_{0}\right)+\varepsilon$. Choose $x, y \in B_{X}$ such that, if $z=\frac{1}{2}(x+y), V\left(z ; \alpha_{m}\left(s_{0}\right)+\frac{\varepsilon}{4}\right) \nsubseteq D\left(x, y ; s_{0}\right)$. By hypothesis, if $s>s_{0}$, we have $V\left(z ; \alpha_{m}\left(s_{0}\right)+\varepsilon\right) \subseteq D(x, y ; s)$. Clearly the distance $d\left(D(x, y ; s), D\left(x, y ; s_{0}\right)\right) \rightarrow 0$ when $s \rightarrow s_{0}^{+}$. Since $D\left(x, y ; s_{0}\right)$ is norm closed (is $w^{*}$-compact), we conclude that $\bigcap_{s>s_{0}} D(x, y ; s)=D\left(x, y ; s_{0}\right)$. Therefore $V\left(z ; \alpha_{m}\left(s_{0}\right)+\varepsilon\right) \subseteq D\left(x, y ; s_{0}\right)$, a contradiction.

1.4. Proposition. Let $X$ be a finite dimensional normed space. The following are equivalent: (a) $B_{X}$ is stable; (b) $B_{X}$ is US.

Proof. As $b \Rightarrow a$ is clear, we prove that $a \Rightarrow b$. If $B_{X}$ is not US, there exist $r>$ 0 and sequences $\left\{x_{n}\right\}_{n \geq 1},\left\{y_{n}\right\}_{n \geq 1}$ in $B_{X}$ such that, if $z_{n}=\frac{1}{2}\left(x_{n}+y_{n}\right)$, then $V\left(z_{n} ; 1 / n\right) \nsubseteq D\left(x_{n}, y_{n} ; r\right)$. Since $B_{X}$ is compact, we can suppose the existence of $\lim _{n \rightarrow \infty} x_{n}=x_{0}, \lim _{n \rightarrow \infty} y_{n}=y_{0}$ and $\lim _{n \rightarrow \infty} z_{n}=z_{0}=\frac{1}{2}\left(x_{0}+y_{0}\right)$. Since $B_{X}$ is stable, there exists $\varepsilon>0$ such that $V\left(z_{0} ; \varepsilon\right) \subseteq D\left(x_{0}, y_{0} ; r / 2\right)$. Taking limits in $V\left(z_{n} ; 1 / n\right) \nsubseteq D\left(x_{n}, y_{n} ; r\right)$, we get a contradiction. 
1.5. Proposition. Let $X$ be a rotund normed space. The following are equivalent:

(a) $X$ is uniformly rotund;

(b) $B_{X}$ is US.

Proof. $(\mathrm{a}) \Rightarrow(\mathrm{b})$. Let $\delta:(0,2] \rightarrow(0,1]$ be a nondecreasing modulus of uniform rotundity of $X$ and take $x, y \in B_{X}, z=(x+y) / 2$ and $r \in(0,2]$. Suppose firstly that $r<\|x-y\|=\varepsilon$. By hypothesis, $B(z ; \delta(\varepsilon)) \subseteq B_{X}$. If $\lambda=r /\left(\delta(\varepsilon)+\frac{1}{2} \varepsilon\right)$, the homothecy $h(y ; \lambda)$, with centre $y$ and ratio $\lambda$, satisfies $h(y ; \lambda)[B(z ; \delta(\varepsilon))]=B_{1} \subseteq V(y ; r)$. Also $h(x ; \lambda)[B(z ; \delta(\varepsilon))]=B_{2} \subseteq$ $V(x ; r)$. Observe that the radius of $B_{i}, i=1,2$, is $\rho(\varepsilon)=[r \cdot \delta(\varepsilon)] /\left[\delta(\varepsilon)+\frac{1}{2} \varepsilon\right]$. In short, if $\varepsilon=\|x-y\|>r$, then $V(z ; \rho(\varepsilon))=B(z ; \rho(\varepsilon))=\frac{1}{2}\left(B_{1}+B_{2}\right) \subseteq$ $D(x, y ; r)$. Suppose finally that $\|x-y\| \leq r$. Then clearly $V(z ; r / 2) \subseteq$ $D(x, y ; r)$. So a modulus of US of $B_{X}$ is

$$
\alpha(r)=\min \left\{\frac{\delta(r) \cdot r}{\delta(r)+r / 2}, \frac{r}{2}\right\}, \quad r \in(0,2] .
$$

(b) $\Rightarrow$ (a). Let $\alpha$ be a modulus of US of $B_{X}$ and $\varepsilon>0$. We prove that there exists $\delta>0$ such that $\|(x+y) / 2\| \leq 1-\delta$, for each $x, y \in S_{X}$ with $\|x-y\| \geq \varepsilon$. By hypothesis $V((x+y) / 2 ; \alpha(\varepsilon / 2)) \subseteq D(x, y ; \varepsilon / 2)$. Since $X$ is rotund, $V(x ; \varepsilon / 2) \cap V(y ; \varepsilon / 2) \subseteq\{(x+y) / 2\}$. But $(x+y) / 2 \notin S_{X}$. Hence $V((x+y) / 2 ; \alpha(\varepsilon / 2)) \cap S_{X}=\varnothing$, because $V((x+y) / 2 ; \alpha(\varepsilon / 2)) \cap S_{X}$ $\subseteq D(x, y ; \varepsilon / 2) \cap S_{X} \subseteq V(x ; \varepsilon / 2) \cap V(y ; \varepsilon / 2) \cap S_{X}=\varnothing$. So the distance $\bar{d}\left((x+y) / 2 ; S_{X}\right) \geq \alpha(\varepsilon / 2)$, that is, $\|(x+y) / 2\| \leq 1-\alpha(\varepsilon / 2)$.

1.6. Proposition. Let $X$ be a normed space, $\alpha$ a modulus of US of $B_{X}$ and $Y \subseteq X$ a 1-complemented subspace of $X$. Then $\alpha$ is a modulus of US of $B_{Y}$.

Proof. Take $\varepsilon>0, x, y \in B_{Y}, z=(x+y) / 2$ and $P: X \rightarrow Y$ a projection with $\|P\|=1$. If the subindex $Y$ indicates we work in $B_{Y}$, we have the following:

$$
V_{Y}(z ; \alpha(\varepsilon))=P(V(z ; \alpha(\varepsilon))) \subseteq P(D(x, y ; \varepsilon))=D_{Y}(x, y ; \varepsilon) .
$$

1.7. Example. Let us see some examples of Banach spaces $X$ such that $B_{X}$ is stable but not US. Indeed, take $X=\left(\sum_{n>1} \bigoplus l_{1+1 / n}\right)_{p}$ or $X=\left(\sum_{n>2} \bigoplus l_{n}\right)_{p}$, $1<p<\infty$. The unit ball $B_{X}$ is rotund but not US, because if $\bar{B}_{X}$ is US, from Proposition 1.6 we would deduce that the family $\left\{l_{n}\right\}_{n \geq 2}$ or $\left\{l_{1+1 / n}\right\}_{n \geq 1}$ is uniformly US (that is, there is a common modulus of US). Now, from the proof of Proposition 1.5, we conclude that these families are uniformly uniformly rotund, which is not true.

1.8. Proposition. Let $X$ be a Banach space and $Y \subseteq X$ a dense US subspace. Then $X$ is US.

Proof. Let $\alpha$ be a left-continuous and nondecreasing modulus of US of $Y$ (if $\alpha$ is not left-continuous, take $\left.\tilde{\alpha}(r)=\lim _{\varepsilon \rightarrow 0^{+}} \alpha(r-\varepsilon)\right)$. We prove that $m \alpha$ is a modulus of US of $B_{X}$ for each $0<m<1$. Pick $x, y \in B_{X}, r \in(0,2]$ and $z \in V((x+y) / 2 ; m \alpha(r))$. Since $\alpha$ is left-continuous, we can choose $x^{\prime} \in V(x ; r) \cap Y, y^{\prime} \in V(y ; r) \cap Y$ and $0<\varepsilon, \delta$ such that

$$
z \in \bar{D}_{Y}\left(x^{\prime}, y^{\prime} ; r-\varepsilon\right), \quad V\left(x^{\prime} ; r-\varepsilon\right) \subseteq V(x ; r-\delta)
$$


and $V\left(y^{\prime} ; r-\varepsilon\right) \subseteq V(y ; r-\delta)$. Now take $x_{1} \in V_{Y}\left(x^{\prime} ; r-\varepsilon\right)$ and $y_{1} \in$ $V_{Y}\left(y^{\prime} ; r-\varepsilon\right)$ such that $z \in \bar{D}_{Y}\left(x_{1}, y_{1} ; \delta / 2\right)$. Next take $x_{2} \in V_{Y}\left(x_{1} ; \delta / 2\right)$ and $y_{2} \in V_{Y}\left(x_{2} ; \delta / 2\right)$ such that $z \in \bar{D}_{Y}\left(x_{2}, y_{2} ; \delta / 4\right)$. By reiteration we get Cauchy sequences $\left\{x_{n}\right\}_{n>1} \subseteq V(x ; r),\left\{y_{n}\right\}_{n>1} \subseteq V(y ; r)$ satisfying $\left\|z-\left(x_{n}+y_{n}\right) / 2\right\| \leq$ $\delta / 2^{n}$. Then, if $x_{0}=\lim _{n \rightarrow \infty} x_{n}$ and $y_{0}=\lim _{n \rightarrow \infty} y_{n}$, clearly $x_{0} \in V(x ; r)$, $y_{0} \in V(y ; r)$ and $z=\left(x_{0}+y_{0}\right) / 2$.

The proof of Proposition 1.9 following is straightforward and left to the reader.

1.9. Proposition. Let $\left\{X_{i}\right\}_{i \in I}$ be a family of Banach spaces.

(A) The following are equivalent $(\mathscr{T} \mathscr{F} \mathscr{A} \mathscr{E})$ :

(1) If $X=\left(\sum_{i \in I} \bigoplus X_{i}\right)_{0}, B_{X}$ is stable; (2) $B_{X_{i}}$ is stable for each $i \in I$.

(B) $\mathscr{T} \mathscr{F} \mathscr{A} \mathscr{E}$ :

(1) If $X=\left(\sum_{i \in I} \bigoplus X_{i}\right)_{\infty}, B_{X}$ is stable.

(2) Each $B_{X_{i}}$ is stable and for each $r \in(0,2]$, there exists a finite subset $F(r) \subseteq I$ such that $\inf \left\{\alpha_{m i}(r): i \in I \backslash F(r)\right\}>0$, where $\alpha_{m i}$ is the maximum modulus of stability of $X_{i}$.

(C) For $p=0$ or $p=\infty, \mathscr{T} \mathscr{F} \mathscr{A} \mathscr{E}$ :

(1) $\alpha$ is a modulus of US of $B_{X}$, with $X=\left(\sum_{i \in I} \bigoplus X_{i}\right)_{p}$.

(2) $\alpha$ is a modulus of US of $B_{X_{i}}$, for each $i \in I$.

(D) Let $I$ be an infinite set, $Y$ a Banach space, $X_{i}=Y$ for each $i \in I$ and $X=\left(\sum_{i \in I} \oplus X_{i}\right)_{\infty} . \mathscr{T} \mathscr{F} \mathscr{A} \mathscr{E}:$

(1) $B_{X}$ is stable, with $X=\left(\sum_{i \in I} \bigoplus X_{i}\right)_{\infty}$.

(2) $B_{Y}$ is US and $\alpha$ is a modulus of US of $B_{Y}$.

(3) $B_{X}$ is US and $\alpha$ is a modulus of US of $B_{X}$.

\section{UNIFORMLY STABLE UNIT BALLS AND INTERSECTION PROPERTY OF BALLS}

A normed space $X$ has the n.2.I.P. (n.2. intersection property, see [6, p. 207; 8; 9]) if for each set of closed balls $S_{1}, S_{2}, \ldots, S_{n}$ in $X$ (with varying centers and radii) such that $S_{i} \cap S_{j} \neq \varnothing$ for all $i$ and $j$, it follows that $\bigcap_{i=1}^{n} S_{i} \neq \varnothing$. It is known (see [4, p. 195]) that if $X$ is 3.2.I.P, then $B_{X}$ is stable and we see here that $\alpha(r)=r / 2$ is a modulus of US of $X$.

2.1. Proposition. Let $X$ be a Banach space with 3.2.I.P. Then $\alpha(r)=r / 2$, $r \in(0,2]$, is a modulus of US of $X$.

Proof. Take $r \in(0,2], x, y \in B_{X}$ and $d \in X$ satisfying $\|d\| \leq r / 2$ and $\|x+y+2 d\| \leq 2$. Let $z=(x+y) / 2$ and $u=z+d$. Then $u \in V(z ; r / 2)$. We must find $x^{\prime} \in V(x ; r)$ and $y^{\prime} \in V(y ; r)$ such that $u=\left(x^{\prime}+y^{\prime}\right) / 2$. Let $B_{1}=B(0 ; 1), B_{2}=B(x+d ; r / 2)$ and $B_{3}=B(x+y+2 d ; 1)$. These balls intersect mutually. So there is $x^{\prime} \in \bigcap_{i=1}^{3} B_{i}$. Now $x^{\prime} \in V(x ; r)$ (since $\left.\left\|x^{\prime}\right\| \leq 1\right), B_{2} \subseteq B(x ; r)$ and, if $y^{\prime}=2 u-x^{\prime}$, also $y^{\prime} \in V(y ; r)$. Finally $u=\left(x^{\prime}+y^{\prime}\right) / 2$.

\section{UNIFORMLY STABLE UNIT BALLS AND BIDUAL}

Let $X$ be a Banach space. We prove that $B_{X}$ is US iff $B_{X^{* *}}$ is US. Let $N \subseteq X$ be a closed subspace, $Y=X / N$ and $Q: X \rightarrow Y$ the quotient map. 
We say that $Q$ is an open quotient if $Q\left(B_{X}\right)=B_{Y}$ and $Q$ restricted to $B_{X}$ is open. We say that $Q$ is uniformly open quotient (u.o.q.) if $Q$ is open and there exist a map $c:(0,2] \rightarrow(0,2]$, called an u.o.q. modulus, such that, for each $x \in B_{X}$ and $r \in(0,2], V(Q(x) ; c(r)) \subseteq Q(V((x ; r)))$.

3.1. Examples. (1) Let $K, K_{1}$ be compact Hausdorff spaces such that $K_{1} \subseteq$ $K$. Then the restriction map $Q: C(K) \rightarrow C\left(K_{1}\right)$, defined by $Q(f)=f_{\mid K_{1}}$ for each $f \in C\left(K_{1}\right)$, is an u.o.q. with modulus $c(r)=r$.

(2) Let $I$ be a set, $\mathscr{U}$ an ultrafilter on $I,\left\{X_{i}\right\}_{i \in I}$ a family of Banach spaces, $Y=\left(\sum_{i \in I} \oplus X_{i}\right)_{\infty}$ and $\left(X_{i}\right)_{\mathscr{U}}$ the ultraproduct with respect to $\mathscr{U}$ (see [10, p. 121]), that is, $\left(X_{i}\right)_{\mathscr{U}}=Y / N$, where $N=\left\{x=\left(x_{i}\right)_{i \in I} \in Y: \lim _{\mathscr{U}} x_{i}=0\right\}$. Then the quotient $Q: Y \rightarrow Y / N$ is an u.o.q. with modulus $c(r)=r$. Indeed, denote by $\left(x_{i}\right)$ an element of $Y$ and $Q\left(\left(x_{i}\right)\right)=\left(x_{i}\right)_{\mathscr{U}}$ the image element in $Y / N$. Suppose that $\left\|\left(x_{i}\right)_{\mathscr{U}}\right\| \leq 1$. Define $\left(y_{i}\right) \in Y$ as follows: $y_{i}=x_{i}$, if $\left\|x_{i}\right\| \leq 1$, and $y_{i}=x_{i} /\left\|x_{i}\right\|$, if $\left\|x_{i}\right\|>1$. Then $\left(y_{i}\right)_{\mathscr{U}}=\left(x_{i}\right)_{\mathscr{U}}$ and $\left(y_{i}\right) \in B_{Y}$. This proves that $Q\left(B_{Y}\right)=B_{Y / N}$. Now take $\left(x_{i}\right) \in B_{Y}, r \in(0,2]$ and $\left(y_{i}\right)_{\mathscr{U}} \in V\left(\left(x_{i}\right)_{\mathscr{U}} ; r\right)$. We want to find $\left(z_{i}\right) \in V\left(\left(x_{i}\right) ; r\right)$ such that $\left(z_{i}\right)_{\mathscr{U}}=\left(y_{i}\right)_{\mathscr{U}}$. We can suppose that $1 \geq\left\|\left(y_{i}\right)\right\|=\sup \left\{\left\|y_{i}\right\|: i \in I\right\} \quad$ if $1<\left\|\left(y_{i}\right)\right\|$ for some $i \in I$, take $\left(y_{i}^{\prime}\right)$ defined by $y_{i}^{\prime}=y_{i}$, if $\left\|y_{i}\right\| \leq 1$, and $y_{i}^{\prime}=y_{i} /\left\|y_{i}\right\|$ if $\left\|y_{i}\right\|>1$, that satisfies $\left\|\left(y_{i}^{\prime}\right)\right\| \leq 1$ and $\left.\left(y_{i}\right)_{\mathscr{U}}=\left(y_{i}^{\prime}\right)_{\mathscr{U}}\right)$. Choose $\left(z_{i}\right)$ as follows: $z_{i}=y_{i}$ if $\left\|x_{i}-y_{i}\right\| \leq r$ and $z_{i}=x_{i}+r \cdot\left(y_{i}-x_{i}\right) /\left\|y_{i}-x_{i}\right\|$ if $\left\|x_{i}-y_{i}\right\|>r$. This proves that $V\left(\left(x_{i}\right)_{\mathscr{U}} ; r\right) \subseteq Q\left(V\left(\left(x_{i}\right) ; r\right)\right)$.

3.2. Proposition. Let $X$ be a Banach space, $N \subseteq X$ a closed subspace and $Q: X \rightarrow Y=X / N$ the quotient map. Then

(a) If $B_{X}$ is stable and $Q$ is an open quotient, $B_{Y}$ is stable.

(b) If $\alpha$ is a modulus of US of $B_{X}$ and $Q$ is an u.o.q. with modulus $c$, then $c \circ \alpha$ is a modulus of US of $B_{Y}$.

Proof. (a) Take $x, y \in B_{X}$ and $r>0$. We prove that there exists $s>0$ such that $V(Q((x+y) / 2) ; s) \subseteq D(Q(x), Q(y) ; r)$. Since $B_{X}$ is stable, there exists $s^{\prime}>0$ such that $V\left((x+y) / 2 ; s^{\prime}\right) \subseteq D(x, y ; r)$. As $Q$ is open, there exists $s>0$ such that $V(Q((x+y) / 2) ; s) \subseteq Q\left[V\left((x+y) / 2 ; s^{\prime}\right)\right]$. In consequence

$$
\begin{aligned}
V\left(Q\left(\frac{x+y}{2}\right) ; s\right) & \subseteq Q\left[V\left(\frac{x+y}{2} ; s\right)\right] \\
& \subseteq Q(D(x, y ; r)) \subseteq D(Q(x), Q(y) ; r) .
\end{aligned}
$$

(b) Let $x, y \in B_{X}$ and $r \in(0,2]$. By hypothesis $V((x+y) / 2 ; \alpha(r)) \subseteq$ $D(x, y ; r)$. Hence:

$$
\begin{aligned}
V\left(Q\left(\frac{x+y}{2}\right) ; c \circ \alpha(r)\right) & \subseteq Q\left[V\left(\frac{x+y}{2} ; \alpha(r)\right)\right] \subseteq Q(D(x, y ; r)) \\
& \subseteq D(Q(x), Q(y) ; r) .
\end{aligned}
$$

So $c \circ \alpha$ is a modulus of US of $Y$.

3.3. Corollary. Let $I$ be a set, $\mathscr{U}$ an ultrafilter on $I, \alpha$ a modulus of US common to all members of the family of Banach spaces $\left\{X_{i}\right\}_{i \in I}$ and $\left(X_{i}\right)_{\mathscr{U}}$ the ultraproduct with respect to $\mathscr{U}$. Then $\alpha$ is a modulus of $U S$ of $B_{\left(X_{i}\right)}$. 
Proof. By (C) of Proposition 1.9, $\alpha$ is a modulus of US of $B_{Y}$, being $Y=$ $\left(\sum_{i \in I} \oplus X_{i}\right)_{\infty}$. Now apply Proposition 3.2 and Example 3.1.

3.4. Proposition. Let $X$ be a Banach space. Then $B_{X}$ is US iff $B_{X^{* *}}$ is US. Moreover, the maximum moduli $\alpha_{m}, \alpha_{m}^{\prime \prime}$ of US of $B_{X}, B_{X^{* *}}$, respectively, satisfy $\alpha_{m}^{\prime \prime}(r)=\lim _{\varepsilon \rightarrow 0^{+}} \alpha_{m}(r+\varepsilon)$, for each $r \in(0,2)$.

Proof. (1) Let $\alpha$ be a modulus of US of $B_{X^{* *}}, x, y \in B_{X}, r \in(0,2], \varepsilon>$ 0 and $d \in X$ such that $\|d\| \leq \alpha(r)$ and $x+y+2 d=u \in B(0 ; 2)$, that is, $u / 2 \in V((x+y) / 2 ; \alpha(r))$. We claim that there exist $x^{\prime} \in V(x ; r+\varepsilon)$, $y^{\prime} \in V(y ; r+\varepsilon)$ such that $x^{\prime}, y^{\prime} \in X$ and $u / 2=\left(x^{\prime}+y^{\prime}\right) / 2$. This fact will imply that $V((x+y) / 2 ; \alpha(r)) \subseteq D(x, y ; r+\varepsilon)$ in $X$. By hypothesis, there exist $z \in V^{\prime \prime}(x ; r), v \in V^{\prime \prime}(y ; r)$ (we put $V^{\prime \prime}$ when we work in $X^{* *}$ ) such that $u / 2=(z+v) / 2$. This implies that $z \in\left[B^{\prime \prime}(0 ; 1) \cap B^{\prime \prime}(x ; r) \cap\right.$ $\left.B^{\prime \prime}(x+2 d ; r) \cap B^{\prime \prime}(u ; 1)\right]$, where $B^{\prime \prime}$ indicates that we take balls in $X^{* *}$. Let $A$ be the subspace of $X^{* *}$ spanned by $\{x, x+2 d, u, z\}$ and $\theta>0$ such that

$$
\begin{aligned}
(1+\theta)\|z\| & \leq 1+\alpha(\varepsilon / 16) \geq(1+\theta)\|z-u\|, \\
(1+\theta)\|x-z\| & \leq r+\varepsilon / 2 \geq\|x+2 d-z\|(1+\theta) .
\end{aligned}
$$

By the principle of local reflexivity (see [10, p. 196]), there exists an operator $T: A \rightarrow X$ such that: (i) $T(w)=w, w \in A \cap X$; (ii) $\|T\| \leq 1+\theta$. So if $z_{1}=T(z)$, we have

$z_{1} \in[B(0 ; 1+\alpha(\varepsilon / 16)) \cap B(x ; r+\varepsilon / 2) \cap B(x+2 d ; r+\varepsilon / 2) \cap B(u ; 1+\alpha(\varepsilon / 16))]$.

Let

$$
\begin{gathered}
x_{1}= \begin{cases}z_{1} & \text { if } z_{1} \in B(0 ; 1), \\
z_{1} /\left\|z_{1}\right\| & \text { if } z_{1} \notin B(0 ; 1),\end{cases} \\
y_{1}= \begin{cases}u-z_{1} & \text { if } z_{1} \in B(u ; 1), \\
\left(u-z_{1}\right) /\left\|u-z_{1}\right\| & \text { if } z_{1} \notin B(u ; 1),\end{cases}
\end{gathered}
$$

and $d_{1} \in X$ such that $x_{1}+y_{1}+2 d_{1}=u$. As $\left\|z_{1}-x_{1}\right\| \leq \alpha(\varepsilon / 16) \geq \| x_{1}+$ $2 d_{1}-z_{1} \|$, we get $\left\|d_{1}\right\| \leq \alpha(\varepsilon / 16)$. Apply again the principle of local reflexivity: there exists $z_{2} \in X$ such that

$$
\begin{aligned}
z_{2} \in[B(0 ; 1+\alpha(\varepsilon / 32)) & \cap B\left(x_{1} ; \varepsilon / 16+\varepsilon / 32\right) \\
\cap B\left(x_{1}+2 d_{1} ; \varepsilon / 16+\varepsilon / 32\right) & \cap B(u ; 1+\alpha(\varepsilon / 32))] .
\end{aligned}
$$

Now $\left\|z_{1}-z_{2}\right\| \leq\left\|z_{1}-x_{1}\right\|+\left\|x_{1}-z_{2}\right\| \leq \alpha(\varepsilon / 16)+\varepsilon(1 / 16+1 / 32) \leq \varepsilon / 4$, because always $\alpha(s) \leq s$. Define $x_{2}, y_{2}$ as in $(*)$ using now $z_{2}$ and take $d_{2} \in X$ such that $x_{2}+y_{2}+2 d_{2}=u$. Then $\left\|d_{2}\right\| \leq \alpha(\varepsilon / 32)$. Apply the principle of local reflexivity: there exists $z_{3} \in X$ such that

$$
\begin{aligned}
z_{3} \in[B(0 ; 1+\alpha(\varepsilon / 64)) & \cap B\left(x_{2} ; \varepsilon\left(2^{-5}+2^{-6}\right)\right) \\
\cap B\left(x_{2}+2 d_{2} ; \varepsilon\left(2^{-5}+2^{-6}\right)\right) & \left.\cap B\left(u ; 1+\alpha\left(\varepsilon \cdot 2^{-6}\right)\right)\right]
\end{aligned}
$$

and $\left\|z_{2}-z_{3}\right\| \leq\left\|z_{2}-x_{2}\right\|+\left\|x_{2}-z_{3}\right\| \leq \alpha\left(\varepsilon \cdot 2^{-5}\right)+\varepsilon\left(2^{-5}+2^{-6}\right) \leq \varepsilon \cdot 2^{-3}$. By reiteration, we obtain a Cauchy sequence $\left\{z_{n}\right\}_{n \geq 1}$ such that, if $x^{\prime}=\lim _{n \rightarrow \infty} z_{n}$, then clearly

$$
x^{\prime} \in[B(0 ; 1) \cap B(x ; r+\varepsilon) \cap B(x+2 d ; r+\varepsilon) \cap B(u ; 1)] .
$$


Thus $x^{\prime} \in V(x ; r+\varepsilon)$ and, if $y^{\prime}=u-x^{\prime}$, then $y^{\prime} \in V(y ; r+\varepsilon)$ and $\frac{u}{2}=$ $\frac{1}{2}\left(x^{\prime}+y^{\prime}\right)$. This proves, in particular, that $\alpha_{m}(r+\varepsilon) \geq \alpha_{m}^{\prime \prime}(r)$ for each $r \in(0,2)$ such that $r+\varepsilon \leq 2$.

(2) Let $B_{X}$ be US. It follows from the principle of local reflexivity that $X^{* *}$ is 1-complemented in $(X)_{\mathscr{U}}$ for some ultrafilter $\mathscr{U}$. Applying Corollary 3.3, we get that $\alpha_{m}^{\prime \prime} \geq \alpha_{m}$. Thus $B_{X^{* *}}$ is US.

Finally for $r \in(0,2)$ and $\varepsilon>0$ with $r+\varepsilon \leq 2$, we have: $\alpha_{m}^{\prime \prime}(r+\varepsilon) \geq$ $\alpha_{m}(r+\varepsilon) \geq \alpha_{m}^{\prime \prime}(r)$. Thus, as $\alpha_{m}^{\prime \prime}$ is right-continuous (see Proposition 1.3), we get that $\alpha_{m}^{\prime \prime}(r)=\lim _{\varepsilon \rightarrow 0^{+}} \alpha_{m}(r+\varepsilon)$, for each $r \in(0 ; 2)$.

\section{The unit Ball in $C(K, X)$}

If $K$ is a compact Hausdorff space and $X$ a Banach space, we denote by $C(K, X)$ the Banach space of continuous functions $f: K \rightarrow X$ with the supremum norm. We prove in this section that $B_{C(K, X)}$ is stable (resp. US) iff $B_{X}$ is stable (resp. US).

4.1. Lemma. Let $X$ be a Banach space such that $B_{X}$ is stable, $x, y \in B_{X}$ and $\varepsilon>0$. Then there exist $0<\delta, \eta$ such that, for each $x^{\prime} \in V(x ; \delta)$, $y^{\prime} \in V(y ; \delta)$, we have

$$
V\left(\left(x^{\prime}+y^{\prime}\right) / 2 ; \eta\right) \subseteq D\left(x^{\prime}, y^{\prime} ; \varepsilon\right) .
$$

Proof. Let $\eta_{1}$ such that

$$
V\left((x+y) / 2 ; \eta_{1}\right) \subseteq D(x, y ; \varepsilon / 2)
$$

and

$$
\delta=\min \left\{\varepsilon / 2, \eta_{1} / 2\right\} .
$$

Then, if $x^{\prime} \in V(x ; \delta)$ and $y^{\prime} \in V(y ; \delta)$, we have

$$
V\left((x+y) / 2 ; \eta_{1}\right) \subseteq D(x, y ; \varepsilon / 2) \subseteq D\left(x^{\prime}, y^{\prime} ; \varepsilon\right) .
$$

But

$$
V\left(\left(x^{\prime}+y^{\prime}\right) / 2 ; \eta_{1} / 2\right) \subseteq V\left((x+y) / 2 ; \eta_{1}\right) .
$$

Thus it is enough to take $\eta=\eta_{1} / 2$.

4.2. Proposition. Let $K$ be a compact Hausdorff space and $X$ a Banach space. The following are equivalent: (1) $B_{C(K, X)}$ is stable; (2) $B_{X}$ is stable.

Proof. (1) $\Rightarrow(2)$ As $X$ is 1 -complemented in $C(K, X)$, it is enough to apply Lemma 1.1 .

(2) $\Rightarrow$ (1) Take $f, g \in B_{C(K, X)}, \varepsilon>0$ and the compact $H=\{(f(k), g(k))$ : $k \in K\} \subseteq B_{X} \times B_{X}$. For each $z=(x, y) \in H$ choose $0<\delta_{z}, \eta_{z}$ fulfilling Lemma 4.1 with respect to $z$ and $\frac{\varepsilon}{2}$. If $U\left(z ; \delta_{z}\right)=V\left(x ; \delta_{z}\right) \times V\left(y ; \delta_{z}\right)$, then the interiors of $\left\{U\left(z ; \delta_{z}\right) ; z \in H\right\}$ cover the compact $H$. So there exists a finite subfamily $\left\{U\left(z_{i} ; \delta_{z_{i}}\right): i=1,2, \ldots, n\right\}$ that also cover $H$. Let $\eta=\min \left\{\eta_{z_{i}}: i=1,2, \ldots, n\right\}$. If $h=(f+g) / 2$, we have

$$
V(h(k) ; \eta) \subseteq D(f(k), g(k) ; \varepsilon / 2) \text { in } B_{X} \quad \text { for each } k \in K .
$$

We claim that $V(h ; \eta) \subseteq D(f, g ; \varepsilon)$ in $B_{C(K, X)}$. Indeed, let $p \in V(h ; \eta)$ and consider the functions 
(a) $\phi: B_{X} \rightarrow 2^{B_{X} \times B_{X}} \quad$ (= family of subsets of $\left.B_{X} \times B_{X}\right)$ such that

$$
x \in B_{X}, \quad \phi(x)=\left\{(u, v) \in B_{X} \times B_{X}: \frac{u+v}{2}=x\right\},
$$

(b) $\psi: K \rightarrow 2^{B_{X} \times B_{X}}$ such that $\psi(k)=[\phi \circ p(k)] \cap[V(f(k) ; \varepsilon) \times V(g(k) ; \varepsilon)]$, $k \in K$.

We have the following:

(I) For each $k \in K, \psi(k)$ is a nonempty closed convex set.

Indeed, as $\phi \circ p(k)$ and $V(f(k) ; \varepsilon) \times V(g(k) ; \varepsilon)$ are closed convex, $\psi(k)$ is closed convex. Let see that $\psi(k) \neq \varnothing$. As $p \in V(h ; \eta),\|h-p\| \leq \eta$ and by (1), for each $k \in K, p(k) \in D(f(k), g(k) ; \varepsilon / 2)$. Thus:

(2) for each $k \in K$ there exists $u \in[\phi \circ p(k)] \cap[V(f(k) ; \varepsilon / 2) \times V(g(k) ; \varepsilon / 2)]$. So $u \in \psi(k)$ and $\psi(k) \neq \varnothing$.

(II) $\psi$ is lower semicontinuous.

Let $U=U_{1} \times U_{2}$ be an open subset of $B_{X} \times B_{X}$ and $k_{0} \in K$ such that $\psi\left(k_{0}\right) \cap U \neq \varnothing$. The set $A=\left\{\left(x_{1}, x_{2}\right) \in \psi\left(k_{0}\right):\left\|x_{1}-f\left(k_{0}\right)\right\|\langle\varepsilon\rangle\left\|x_{2}-g\left(k_{0}\right)\right\|\right\}$ is nonempty (by (2)) and dense in $\psi\left(k_{0}\right)$. As $\psi\left(k_{0}\right) \cap U \neq \varnothing$, also $A \cap U \neq \varnothing$. Take $z=\left(z_{1}, z_{2}\right) \in A \cap U$. The continuity of $f$ and $g$ implies the existence of $\rho>0$ and a neighbourhood $G_{1}$ of $k_{0}$ in $K$ such that

(3) for each $k \in G_{1}, V\left(z_{1} ; \rho\right) \times V\left(z_{2} ; \rho\right) \subseteq U \cap[V(f(k) ; \varepsilon) \times V(g(k) ; \varepsilon)]$. As $B_{X}$ is stable and $\left(z_{1}+z_{2}\right) / 2=p\left(k_{0}\right)$, there exists $\delta>0$ satisfying $V\left(p\left(k_{0}\right) ; \delta\right) \subseteq D\left(z_{1}, z_{2} ; \rho\right)$. In consequence:

(4) for each $y \in V\left(p\left(k_{0}\right) ; \delta\right), \phi(y) \cap\left[V\left(z_{1} ; \rho\right) \times V\left(z_{2} ; \rho\right)\right] \neq \varnothing$. Let $G_{2}=p^{-1}\left(V\left(p\left(k_{0}\right) ; \delta\right)\right)$ and $G=G_{1} \cap G_{2}$ a neighbourhood of $k_{0}$ in $K$. Then for each $k \in G$ we have $\psi(k) \cap U \neq \varnothing$. Indeed, take $k \in G$ :

(a) As $k \in G_{2}$, we have, by (4), that $[\phi \circ p(k)] \cap\left[V\left(z_{1} ; \rho\right) \times V\left(z_{2} ; \rho\right)\right] \neq \varnothing$.

(b) As $k \in G_{1}$, we have, by (3), that $V\left(z_{1} ; \rho\right) \times V\left(z_{2} ; \rho\right) \subseteq U \cap[V(f(k) ; \varepsilon) \times$ $V(g(k) ; \varepsilon)]$. Therefore $U \cap \psi(k)=[\phi \circ p(k)] \cap[V(f(k) ; \varepsilon) \times V(g(k) ; \varepsilon)] \cap U \neq$ $\varnothing$. Thus $\psi$ is lower semicontinuous.

By Michael's selection theorem [13, p. 5], $\psi$ admits a continuous selection $\left(s_{1}, s_{2}\right)=s: K \rightarrow B_{X} \times B_{X}$ such that $p=\frac{1}{2}\left(s_{1}+s_{2}\right)$ and $s_{1} \in V(f ; \varepsilon), s_{2} \in$ $V(g ; \varepsilon)$. This proves that $V(h ; \eta) \subseteq D(f, g ; \varepsilon)$ and that $B_{C(K, X)}$ is stable.

4.3. Proposition. Let $K$ be a compact Hausdorff set and $X$ a Banach space. Then $B_{X}$ is US iff $B_{C(K, X)}$ is US and the maximum moduli $\alpha_{m}^{X}, \alpha_{m}^{C(K, X)}$ of $U S$ of $B_{X}$ and $B_{C(K, X)}$, respectively, satisfy

$$
\text { for each } r \in(0,2], \quad \alpha_{m}^{X}(r) \geq \alpha_{m}^{C(K, X)}(r) \geq \lim _{\varepsilon \rightarrow 0^{+}} \alpha_{m}^{X}(r-\varepsilon) .
$$

Proof. As $X$ is 1-complemented in $C(K, X)$, applying Proposition 1.6, we obtain that $B_{X}$ is US, provided that $B_{C(K, X)}$ is US and that $\alpha_{m}^{X} \geq \alpha_{m}^{C(K, X)}$.

Suppose now that $B_{X}$ is US and that $\alpha$ is a modulus of US of $B_{X}$. Let $f, g \in B_{C(K, X)}, h=(f+g) / 2$ and $r \in(0,2]$. We prove that for each $0<\varepsilon<r, V(h ; \alpha(r-\varepsilon)) \subseteq D(f, g ; r)$ in $B_{C(K, X)}$. Take $p \in V(h ; \alpha(r-\varepsilon))$ and consider the functions $\phi, \psi$ as in Proposition 4.2, that is,

$$
x \in B_{X}, \quad \phi(x)=\left\{(u, v) \in B_{X} \times B_{X}: \frac{u+v}{2}=x\right\} ;
$$




$$
k \in K, \quad \psi(k)=[\phi \circ p(k)] \cap[V(f(k) ; r) \times V(g(k) ; r)] .
$$

As in Proposition 4.2, $\psi$ is lower semicontinuous and $\psi(k)$ is a closed convex nonempty set, for each $k \in K$. Applying Michael's selection theorem [13, p. 5], we obtain that $p \in D(f, g ; r)$ in $B_{C(K, X)}$. Thus $B_{C(K, X)}$ is US and, for each $r \in(0,2], \alpha_{m}^{C(K, X)}(r) \geq \lim _{\varepsilon \rightarrow 0^{+}} \alpha_{m}^{X}(r-\varepsilon)$.

\section{The Unit Ball in $A(K, X)$}

If $K$ is a compact convex set and $X$ a Banach space, $A(K, X)$ will be the Banach space of affine continuous functions $f: K \rightarrow X$ with the supremum norm. A compact convex set $K$ is a Choquet simplex iff $A(K, X)$ is a $L_{1}$ predual (see [6, p. 183]; [1, p. 84]).

5.1. Proposition. Let $K$ be a Choquet simplex and $X$ a Banach space. $\mathscr{T} \mathscr{F} \mathscr{A} \mathscr{E}$ :

(1) $B_{A(K, X)}$ is stable; (2) $B_{X}$ is stable.

Proof. (1) $\Rightarrow(2)$ As $X$ is 1-complemented in $A(K, X)$, this follows from Lemma 1.1.

(2) $\Rightarrow$ (1) Take $f, g \in B_{A(K, X)}, \varepsilon>0$ and $h=(f+g) / 2$. From Lemma 4.1 it follows that there exists $\eta>0$ such that, for each $k \in K, V(h(k) ; \eta) \subseteq$ $D(f(k), g(k) ; \varepsilon / 2)$ in $B_{X}$. We claim that $V(h ; \eta) \subseteq D(f, g ; \varepsilon)$ in $B_{A(K, X)}$. Indeed, pick $p \in V(h ; \eta)$ and consider the functions $\phi, \psi$ of Proposition 4.2. We know that $\psi$ is lower semicontinuous and that $\psi(k)$ is a convex closed nonempty set, for each $k \in K$. Moreover, it is easily proved that $\psi$ is affine, that is, if $k_{1}, k_{2} \in K, 0 \leq \lambda \leq 1$, and $k=\lambda k_{1}+(1-\lambda) k_{2}$, then $\lambda \psi\left(k_{1}\right)+(1-\lambda) \psi\left(k_{2}\right) \subseteq \psi\left(\lambda k_{1}+(1-\lambda) k_{2}\right)$. So applying Lazar's theorem [7, Theorem 3.1, p. 511], we get an affine continuous selection $s=\left(s_{1}, s_{2}\right)$ : $K \rightarrow B_{X} \times B_{X}$ such that $p=\frac{1}{2}\left(s_{1}+s_{2}\right), s_{1} \in V(f ; \varepsilon)$ and $s_{2} \in V(g ; \varepsilon)$. Therefore $V(h ; \eta) \subseteq D(f, g ; \varepsilon)$.

5.2. Proposition. Let $K$ be a Choquet simplex and $X$ a Banach space. Then $B_{X}$ is US iff $B_{A(K, X)}$ is US, and the maximum moduli $\alpha_{m}^{X}, \alpha_{m}^{A(K, X)}$ of US of $B_{X}$ and $B_{A(K, X)}$, respectively, satisfy

$$
\text { for each } r \in(0,2], \quad \alpha_{m}^{X}(r) \geq \alpha_{m}^{A(K, X)}(r) \geq \lim _{\varepsilon \rightarrow 0^{+}} \alpha_{m}^{X}(r-\varepsilon) .
$$

Proof. This proof is similar to the proof of Proposition 4.3, applying Lazar's theorem instead of Michael's theorem.

Open problem. Let $K$ be a compact convex set satisfying Proposition 5.1 or Proposition 5.2. Is $K$ a Choquet simplex?

\section{THE NORM IN $L_{p}(\mu, X)$}

In this section we study the unit ball $B_{L_{p}(\mu, X)}$ when the unit ball $B_{X}$ is stable. Let us begin with $L_{\infty}(\mu, X)$. 
6.1. Proposition. Let $X$ be a Banach space and $(\Omega, \Sigma, \mu)$ a measure space.

(A) Suppose that $\mu$ is purely atomic with a finite number of atoms. Then

(1) $B_{L_{\infty}(\mu, X)}$ is stable iff $B_{X}$ is stable.

(2) $\alpha$ is a modulus of US of $B_{L_{\infty}(\mu, X)}$ iff $\alpha$ is a modulus of US of $B_{X}$.

(B) Suppose that the measure space $(\Omega, \Sigma, \mu)$ does not reduce to a purely atomic measure with a finite number of atoms. Then $\mathscr{T} \mathscr{F} \mathscr{A} \mathscr{E}$ :

(a) $B_{L_{\infty}(\mu, X)}$ is stable.

(b) $B_{X}$ is US.

(c) $B_{L_{\infty}(\mu, X)}$ is US.

Proof. (A) In this case $L_{\infty}(\mu, X)$ is isometric to $\left(\sum_{i=1}^{n} \bigoplus X_{i}\right)_{\infty}$, with $X_{i}=$ $X, i=1,2, \ldots, n$, and $n$ the number of atoms of $(\Omega, \Sigma, \mu)$. Now apply Proposition 1.9.

(B) (a) $\Rightarrow$ (b) Apply that $\left(\sum_{n>1} \oplus X_{n}\right)_{\infty}$, with $X_{n}=X$, is 1-complemented in $L_{\infty}(\mu, X)$ and Proposition 1.9.

(b) $\Rightarrow$ (c) Let $Y \subseteq L_{\infty}(\mu, X)$ be the subspace of bounded countable $X$ valued functions $f=\sum_{n>1} x_{n} \cdot \chi_{A_{n}}, A_{n} \in \Sigma, A_{n} \cap A_{m}=\varnothing$ if $n \neq m$. As $Y$ is dense in $L_{\infty}(\mu, \bar{X})$, by Proposition 1.8 , it is enough to prove that $Y$ is US. But this follows immediately from (D) of Proposition 1.9 and the fact that, if $f_{i} \in Y, i=1,2, \ldots, m$, there is in $Y$ an isometric copy of $Z=\left(\sum_{n \geq 1} \bigoplus X_{n}\right)_{\infty}, X_{n}=X, n \geq 1$, such that $f_{i} \in Z$.

(c) $\Rightarrow$ (a) This is immediate.

In the following we prove that $B_{L_{p}(\mu, X)}, 1 \leq p<\infty$, is stable iff $B_{X}$ is stable. As $X$ is 1 -complemented in $L_{p}(\mu, X)$, it is enough to prove that $B_{L_{p}(\mu, X)}$ is stable when $B_{X}$ is stable. We begin with the case $1<p<\infty$.

6.2. Lemma. Let $X$ be a Banach space with stable unit ball $B_{X}, K$ a compact Hausdorff space, $f_{i} \in C(K, X), i=0,1,2$, such that $f_{0}=\frac{1}{2}\left(f_{1}+f_{2}\right)$ and $\left\|f_{0}(k)\right\|=\left\|f_{1}(k)\right\|=\left\|f_{2}(k)\right\|$ for each $k \in K$, and $\varepsilon>0$. Then there exists $\eta>0$ such that for each $g \in B\left(f_{0} ; \eta\right)$ we can choose $g_{i} \in B\left(f_{i} ; \varepsilon\right), i=1,2$, satisfying $g=\frac{1}{2}\left(g_{1}+g_{2}\right)$ and $\|g(k)\|=\left\|g_{1}(k)\right\|=\left\|g_{2}(k)\right\|$ for each $k \in K$.

Proof. Define $\phi: X \rightarrow 2^{X \times X}$ by $\phi(x)=\{(u, v) \in X \times X: x=(u+v) / 2,\|x\|=$ $\|u\|=\|v\|\}, x \in X$.

(1) Let us see that $\phi$ is lower semicontinuous. Pick $\delta>0, x_{0} \in X$ and $\left(x_{1}, x_{2}\right) \in \phi\left(x_{0}\right)$. We prove that there exists $\rho>0$ (depending on $\left.\delta, x_{0}, x_{1}, x_{2}\right)$ such that each $y_{0} \in B\left(x_{0} ; \rho\right)$ satisfies $\phi\left(y_{0}\right) \cap\left[B\left(x_{1} ; \delta\right) \times\right.$ $\left.B\left(x_{2} ; \delta\right)\right] \neq \varnothing$. If $x_{0}=0$, take $\rho=\delta$. Assume that $x_{0} \neq 0$ and, without loss of generality, that $\left\|x_{0}\right\|=1$. Since $B_{X}$ is stable, there exists $0<\rho^{\prime}<1$ such that, for each $x_{0}^{\prime} \in B\left(x_{0} ; \rho^{\prime}\right) \cap S_{X}$, there exists $x_{i}^{\prime} \in B\left(x_{i} ; \delta / 2\right) \cap S_{X}, i=1,2$, satisfying $x_{0}^{\prime}=\frac{1}{2}\left(x_{1}^{\prime}+x_{2}^{\prime}\right)$. Put $\rho=\min \left\{\rho^{\prime} / 2, \delta / 2\right\}$ and let $y_{0} \in B\left(x_{0} ; \rho\right)$ and $x_{0}^{\prime}=y_{0} /\left\|y_{0}\right\|$. As $\left\|x_{0}^{\prime}-x_{0}\right\| \leq\left\|x_{0}^{\prime}-y_{0}\right\|+\left\|y_{0}-x_{0}\right\| \leq 2 \rho \leq \rho^{\prime}$, there exist $x_{i}^{\prime} \in B\left(x_{i} ; \delta / 2\right) \cap S_{X}, i=1,2$, with $x_{0}^{\prime}=\frac{1}{2}\left(x_{1}^{\prime}+x_{2}^{\prime}\right)$. Put $y_{i}=\left\|y_{0}\right\|$. $x_{i}^{\prime}, i=1,2$. Then $\left(y_{1}, y_{2}\right) \in \phi\left(y_{0}\right)$ and $\left\|x_{i}-y_{i}\right\| \leq\left\|x_{i}-x_{i}^{\prime}\right\|+\left\|x_{i}^{\prime}-y_{i}\right\| \leq$ $\delta / 2+\delta / 2=\delta, i=1,2$, that is, $\phi\left(x_{0}\right) \cap\left[B\left(x_{1} ; \delta\right) \times B\left(x_{2} ; \delta\right)\right] \neq \varnothing$.

(2) Let $f_{i} \in C(K, X), i=0,1,2$, and $\varepsilon>0$ satisfy the statement of Lemma 6.2. We prove that there exists $\eta>0$ such that $\phi(x) \cap\left[B\left(f_{1}(k) ; \varepsilon / 2\right) \times\right.$ $\left.B\left(f_{2}(k) ; \varepsilon / 2\right)\right] \neq \varnothing$, provided that $k \in K$ and $x \in B\left(f_{0}(k) ; \eta\right)$. By (1), 
for each $k \in K$, there exists $\eta_{k}>0$ satisfying, for $x \in B\left(f_{0}(k) ; \eta_{k}\right)$, that $\phi(x) \cap\left[B\left(f_{1}(k) ; \varepsilon / 4\right) \times B\left(f_{2}(k) ; \varepsilon / 4\right)\right] \neq \varnothing$. For $k \in K$ put

$$
G_{k}=f_{0}^{-1}\left[B\left(f_{0}(k) ; \eta_{k} / 2\right)\right] \cap f_{1}^{-1}\left[B\left(f_{1}(k) ; \varepsilon / 4\right)\right] \cap f_{2}^{-1}\left[B\left(f_{2}(k) ; \varepsilon / 4\right)\right] .
$$

The family of neighbourhoods $\left\{G_{k}\right\}_{k \in K}$ cover $K$. So there is a finite family $\left\{k_{1}, \ldots, k_{n}\right\} \subseteq K$ such that $\left\{G_{k_{i}}: i=1,2, \ldots, n\right\}$ also cover $K$. Let $\eta=\min \left\{\eta_{k} / 2: k=1, \ldots, n\right\}$. If $k \in K$ and $x \in B\left(f_{0}(k) ; \eta\right)$, there exists $i_{0} \in\{1, \ldots, n\}$, for example, $i_{0}=1$, such that $k \in G_{k_{1}}$ and $f_{0}(k) \in$ $B\left(f_{0}\left(k_{1}\right) ; \frac{1}{2} \eta_{k_{1}}\right)$. Thus $x \in B\left(f_{0}\left(k_{1}\right) ; \eta_{k_{1}}\right)$ and there exists $\left(x_{1}, x_{2}\right) \in \phi(x) \cap$ $\left[B\left(f_{1}\left(k_{1}\right) ; \varepsilon / 4\right) \times B\left(f_{2}\left(k_{1}\right) ; \varepsilon / 4\right)\right]$ satisfying

$$
\left\|x_{i}-f_{i}(k)\right\| \leq\left\|x_{i}-f_{i}\left(k_{1}\right)\right\|+\left\|f_{i}\left(k_{1}\right)-f_{i}(k)\right\| \leq \frac{\varepsilon}{4}+\frac{\varepsilon}{4}=\frac{\varepsilon}{2}, \quad i=1,2,
$$

that is, $\left(x_{1}, x_{2}\right) \in \phi(x) \cap\left[B\left(f_{1}(k) ; \varepsilon / 2\right) \times B\left(f_{2}(k) ; \varepsilon / 2\right)\right]$.

(3) Pick $g \in C(K, X)$ such that $\left\|g-f_{0}\right\| \leq \eta$ and define $\psi: K \rightarrow 2^{X \times X}$ as follows:

$$
\psi(k)=[\phi \circ g(k)] \cap\left[B\left(f_{1}(k) ; \varepsilon\right) \times B\left(f_{2}(k) ; \varepsilon\right)\right], \quad k \in K .
$$

As in Proposition 4.2, it is proved that $\psi$ is lower semicontinuous and that $\psi(k)$ is a nonempty closed convex set for each $k \in K$. Applying Michael's theorem [13, p. 5], there exists a continuous selection $s=\left(s_{1}, s_{2}\right): K \rightarrow X \times X$ such that $s(k) \in \psi(k)$ for each $k \in K$. Now take $g_{i}=s_{i}, i=1,2$.

6.3. Proposition. Let $X$ be a Banach space with $B_{X}$ stable, $K$ a compact Hausdorff space, $\mu$ a positive Radon measure on $K$ and $1<p<\infty$. Then $B_{L_{p}(\mu, X)}$ is stable.

Proof. Let $0<\varepsilon \leq 1$ and $f_{i} \in B_{L_{p}(\mu, X)}, i=0,1,2$, such that $f_{0}=\frac{1}{2}\left(f_{1}+f_{2}\right)$. We prove that there exists $\rho>0$ such that $V\left(f_{0} ; \rho\right) \subseteq D\left(f_{1}, f_{2} ; \varepsilon\right)$, assuming $\left\|f_{i}\right\|=1, i=0,1,2$ (if $\left\|f_{0}\right\|<1$, this fact is clearly true). As $1<p<\infty$, we have $\left\|f_{0}(k)\right\|=\left\|f_{1}(k)\right\|=\left\|f_{2}(k)\right\|, k \in K$, almost everywhere (a.e.). Let $G=\left\{x \in X \backslash\{0\}: x /\|x\| \notin \operatorname{Ext}\left(B_{X}\right)\right\}$. Note that $G$ is open because $B_{X}$ is stable and $\operatorname{Ext}\left(B_{X}\right)$ is closed. If $U=f_{0}^{-1}(G)$, then $f_{1}(k)=f_{0}(k)=f_{2}(k)$, $k \in K \backslash U$ a.e. If $\mu(U)=0$, there is nothing to prove. Suppose that $\mu(U)>0$ and choose a compact subset $K_{0} \subseteq U$ such that $\mu\left(K_{0}\right)>0, f_{i}$ continuous on $K_{0}, i=0,1,2$, and $\left[\int_{K \backslash K_{i}}\left\|f_{i}-f_{0}\right\|^{p} \cdot d \mu\right]^{1 / p} \leq \varepsilon / 8, i=1,2$. Let $\delta=(\varepsilon / 4) /\left[\mu\left(K_{0}\right)\right]^{1 / p}$. By Lemma 6.2 , there exists $0<\eta<1$ such that, if $h \in C\left(K_{0}, X\right)$ and $\left\|h-f_{0}\right\|_{K_{0}} \leq \eta\left(\|\cdot\|_{K_{0}}\right.$ is the supremum norm on $\left.K_{0}\right)$, then there exist $h_{i} \in C\left(K_{0}, X\right)$ satisfying $\left\|h_{i}-f_{i}\right\|_{K_{0}} \leq \delta, i=1,2, h=\frac{1}{2}\left(h_{1}+h_{2}\right)$ and $\|h(k)\|=\left\|h_{1}(k)\right\|=\left\|h_{2}(k)\right\|$ for each $k \in K_{0}$. Let

$$
M=\max \left\{1,\left\|f_{i}\right\|_{K_{0}}: i=0,1,2\right\} \text { and } \rho=(\varepsilon \eta) /(16 M) .
$$

Pick $g \in V\left(f_{0} ; \rho\right)$ in $B_{L_{p}(\mu, X)}$ and let $A=\left\{k \in K_{0}:\left\|f_{0}(k)-g(k)\right\|>\eta\right\}$. Note that

$$
\eta \cdot \mu(A)^{1 / p} \leq\left[\int_{A}\left\|f_{0}(k)-g(k)\right\|^{p} \cdot d \mu\right]^{1 / p} \leq \rho=(\varepsilon \eta) /(16 M)
$$

that is, $\mu(A) \leq[\varepsilon /(16 M)]^{p}$. Now choose a compact subset $K_{1} \subseteq K_{0} \backslash A$ such that $g$ is continuous on $K_{1}$ and $\left[\int_{K_{0} \backslash K_{1}}\left\|f_{i}(k)-f_{0}(K)\right\|^{p} \cdot d \mu\right]^{1 / p} \leq \varepsilon / 4, i=$ 1,2 . Note that this choice is possible because 


$$
\left[\int_{A}\left\|f_{i}(k)-f_{0}(k)\right\|^{p} \cdot d \mu\right]^{1 / p} \leq 2 M \cdot \mu(A)^{1 / p} \leq \varepsilon / 8, \quad i=1,2 .
$$

Let $\hat{g}: K_{0} \rightarrow X$ be a continuous extension of $g_{\mid K_{1}}$ to $K_{0}$ satisfying $\left\|f_{0}-\hat{g}\right\|_{K_{0}} \leq$ $\eta$. By Lemma 6.2, there exists $\hat{g}_{i} \in C\left(K_{0}, X\right)$ such that $\left\|\hat{g}_{i}-f_{i}\right\|_{K_{0}} \leq \delta$, $i=1,2$, and for each $k \in K_{0}, \hat{g}(k)=\frac{1}{2}\left(\hat{g}_{1}(k)+\hat{g}_{2}(k)\right)$ and $\left\|\hat{g}_{1}(k)\right\|=$ $\|\hat{g}(k)\|=\left\|\hat{g}_{2}(k)\right\|$. Define

$$
k \in K, \quad g_{i}(k)=\left\{\begin{array}{ll}
\hat{g}_{i}(k), & k \in K_{1}, \\
g(k), & k \in K \backslash K_{1},
\end{array} \quad i=1,2 .\right.
$$

Then $g=\frac{1}{2}\left(g_{1}+g_{2}\right),\left\|g_{i}\right\|_{p}=\|g\|_{p} \leq 1$ and $\left\|f_{i}-g_{i}\right\|_{p} \leq \varepsilon, i=1,2$, because

$$
\begin{aligned}
\left\|f_{i}-g_{i}\right\|_{p} \leq & {\left[\int_{K_{1}}\left\|f_{i}-g_{i}\right\|^{p} \cdot d \mu\right]^{1 / p}+\left[\int_{K \backslash K_{1}}\left\|f_{i}-f_{0}\right\|^{p} \cdot d \mu\right]^{1 / p} } \\
& +\left[\int_{K \backslash K_{1}}\left\|f_{0}-g\right\|^{p} \cdot d \mu\right]^{1 / p} \\
\leq & \delta \cdot \mu\left(K_{1}\right)^{1 / p}+\frac{\varepsilon}{8}+\frac{\varepsilon}{4}+\rho \leq \varepsilon
\end{aligned}
$$

6.4. Proposition. Let $(\Omega, \Sigma, \mu)$ be a positive measure space, $X$ a Banach space with $B_{X}$ stable and $1<p<\infty$. Then $B_{L_{p}(\mu, X)}$ is stable.

Proof. Let $K$ be the Stone compact of the measure algebra $(\Sigma, \mu)$ and $m$ the induced Radon measure on the Borel sets $\mathrm{Bo}(K)$ (see [6, p. 119]). If $\rho$ is a lifting on $L_{\infty}(\mu)$, we can obtain (see [14]) a map $\tilde{\rho}: \Omega \rightarrow K$ such that the map $F: L_{p}(m, X) \rightarrow L_{p}(\mu, X)$, defined by $F(f)=f \circ \tilde{\rho}$, is an isometry. Now apply Proposition 6.3.

6.5. Proposition. Let $\left\{X_{i}\right\}_{i \in I}$ be a family of Banach spaces with $B_{X_{i}}$ stable and $1<p<\infty$. Then, if $Y=\left(\sum_{i \in I} \bigoplus X_{i}\right)_{p}, B_{Y}$ is stable.

Proof. Let $x, y, z \in S_{Y}$ such that $z=\frac{1}{2}(x+y)$. We can suppose that $I=\mathbb{N}$. As $1<p<\infty$, then $\left\|x_{n}\right\|=\left\|y_{n}\right\|=\left\|z_{n}\right\|, n \geq 1$. Let us prove that, for a given $\varepsilon>0$, there exists $\rho>0$ such that $V(z ; \rho) \subseteq D(x, y ; \varepsilon)$. Choose $m \in \mathbb{N}$ such that $\left[\sum_{n \geq m}\left\|x_{n}-z_{n}\right\|^{p}\right]^{1 / p} \leq \varepsilon / 4 \geq\left[\sum_{n \geq m}\left\|y_{n}-z_{n}\right\|^{p}\right]^{1 / p}$. From the proof of Lemma 6.2 we know that there exist $\eta>0$ such that, if $\left\|z_{n}-z_{n}^{\prime}\right\| \leq \eta$, $n \leq m$, there exist $x_{n}^{\prime}, y_{n}^{\prime} \in X_{n}$ fulfilling $\left\|x_{n}^{\prime}\right\|=\left\|y_{n}^{\prime}\right\|=\left\|z_{n}^{\prime}\right\|, z_{n}^{\prime}=\frac{1}{2}\left(x_{n}^{\prime}+z_{n}^{\prime}\right)$, $\left\|x_{n}^{\prime}-x_{n}\right\| \leq \varepsilon /\left(4 \cdot m^{1 / p}\right) \geq\left\|y_{n}^{\prime}-y_{n}\right\|$. Take $\rho=\min \{\varepsilon / 2, \eta\}$ and $z^{\prime} \in V(z ; \rho)$. Let $x^{\prime}, y^{\prime}$ such that $x_{n}^{\prime}, y_{n}^{\prime}, n \leq m$, are as above and $x_{n}^{\prime}=z_{n}^{\prime}=y_{n}^{\prime}, n>m$. Then $z^{\prime}=\frac{1}{2}\left(x^{\prime}+y^{\prime}\right)$ with $x^{\prime} \in V(x ; \varepsilon)$ and $y^{\prime} \in V(y ; \varepsilon)$.

6.6. Lemma. Let $X$ be a Banach space with $B_{X}$ stable, $K$ a compact Hausdorff space and $f_{i} \in C(K, X), i=0,1,2$, continuous functions such that $f_{0}=\frac{1}{2}\left(f_{1}+f_{2}\right)$ and $\left\|f_{0}(k)\right\|=\frac{1}{2}\left(\left\|f_{1}(k)\right\|+\left\|f_{2}(k)\right\|\right), k \in K$. Then, given $\varepsilon>0$, there exists $\eta>0$ such that, for each $g \in B\left(f_{0} ; \eta\right)$, there exist $g_{i} \in B\left(f_{i} ; \varepsilon\right)$, $i=1,2$, satisfying 


$$
g=\frac{1}{2}\left(g_{1}+g_{2}\right), \quad\|g(k)\|=\frac{1}{2}\left(\left\|g_{1}(k)\right\|+\left\|g_{2}(k)\right\|\right), \quad k \in K .
$$

Proof. Define $\phi: X \rightarrow 2^{X \times X}$ by $\phi(x)=\{(u, v) \in X \times X: x=(u+v) / 2$, $\left.\|x\|=\frac{1}{2}(\|u\|+\|v\|)\right\}, x \in X$.

(1) Let us see that $\phi$ is lower semicontinuous. Take $\varepsilon>0, x_{0} \in X$ and $\left(x_{1}, x_{2}\right) \in \phi\left(x_{0}\right)$. We prove that there exists $\eta>0$ such that, for each $x_{0}^{\prime} \in$ $B\left(x_{0} ; \eta\right), \phi\left(x_{0}^{\prime}\right) \cap\left[B\left(x_{1} ; \varepsilon\right) \times B\left(x_{2} ; \varepsilon\right)\right] \neq \varnothing$. If $x_{i}=\lambda_{i} \cdot x_{0}, \lambda_{i} \geq 0, i=1,2$, take $\eta=\varepsilon / 2$. Suppose that $x_{i} \neq \lambda_{i} \cdot x_{0}$. Without loss of generality, assume that $\left\|x_{2}\right\|=1,\left\|x_{0}\right\|=1-a>0$ and $\left\|x_{1}\right\|=1-2 a>0$. Let $y_{i}=x_{i} /\left\|x_{i}\right\|$, $i=0,1,2$. We claim that $y_{0} \in\left[y_{1}, y_{2}\right] \quad\left(=\left\{\mu y_{1}+(1-\mu) y_{2}: 0 \leq \mu \leq 1\right\}\right)$. Indeed, if $\lambda=(1-2 a) / 2(1-a)$, then

$$
\begin{aligned}
y_{0}= & \frac{x_{0}}{1-a}=\frac{1}{2} \frac{x_{1}}{1-a}+\frac{1}{2} \frac{x_{2}}{1-a}=\frac{1}{2} \frac{1-2 a}{1-a} \frac{x_{1}}{1-2 a} \\
& +\left(1-\frac{1}{2} \frac{1-2 a}{1-a}\right) x_{2}=\lambda y_{1}+(1-\lambda) y_{2} .
\end{aligned}
$$

Thus $\left[y_{1}, y_{2}\right] \subseteq S_{X}$. Observe that, if $0<t<1$ and we define $\phi_{t}: X \rightarrow 2^{X \times X}$ as $\phi_{t}(x)=\{(u, v) \in X \times X: x=t u+(1-t) v,\|x\|=\|u\|=\|v\|\}, x \in X$, then the proof of Lemma 6.2 implies that $\phi_{t}$ is lower semicontinuous. Then, for $\lambda=(1-2 a) / 2(1-a)$, there exists $\eta^{\prime}>0$ such that, for each $y_{0}^{\prime} \in B\left(y_{0} ; \eta^{\prime}\right)$, there exists $y_{i}^{\prime} \in B\left(y_{i} ; \varepsilon\right), i=1,2$, such that

$$
y_{0}^{\prime}=\lambda y_{1}^{\prime}+(1-\lambda) y_{2}^{\prime}, \quad\left\|y_{0}^{\prime}\right\|=\left\|y_{1}^{\prime}\right\|=\left\|y_{2}^{\prime}\right\| .
$$

Let $\eta=(1-a) \eta^{\prime}$ and $x_{0}^{\prime} \in B\left(x_{0} ; \eta\right)$. Then, if $y_{0}^{\prime}=x_{0}^{\prime} /(1-a)$, we have $y_{0}^{\prime} \in B\left(y_{0} ; \eta^{\prime}\right)$. Thus there exist $y_{i}^{\prime} \in B\left(y_{i} ; \varepsilon\right), i=1,2$, satisfying $(*)$. In particular

$$
y_{0}^{\prime}=\frac{x_{0}^{\prime}}{1-a}=\frac{1}{2} \frac{1-2 a}{1-a} y_{1}^{\prime}+\frac{1}{2} \frac{1}{1-a} y_{2}^{\prime} .
$$

Let $x_{1}^{\prime}=y_{1}^{\prime}(1-2 a)$ and $x_{2}^{\prime}=y_{2}^{\prime}$. Then

$$
\begin{aligned}
x_{0}^{\prime} & =\frac{1}{2} x_{1}^{\prime}+\frac{1}{2} x_{2}^{\prime}, \\
\left\|x_{0}^{\prime}\right\| & =(1-a)\left\|y_{0}^{\prime}\right\|=(1-a) \frac{1}{2}\left(\left\|y_{1}^{\prime}\right\|+\left\|y_{2}^{\prime}\right\|\right) \\
& =\frac{1}{2}\left[(1-2 a)\left\|y_{1}^{\prime}\right\|+(1-a+a)\left\|y_{2}^{\prime}\right\|\right]=\frac{1}{2}\left(\left\|x_{1}^{\prime}\right\|+\left\|x_{2}^{\prime}\right\|\right) .
\end{aligned}
$$

Moreover $\left\|x_{i}-x_{i}^{\prime}\right\| \leq \varepsilon$, that is, $\left(x_{1}^{\prime}, x_{2}^{\prime}\right) \in \phi\left(x_{0}^{\prime}\right) \cap\left[B\left(x_{1} ; \varepsilon\right) \times B\left(x_{2} ; \varepsilon\right)\right]$. Thus $\phi$ is lower semicontinuous.

(2) Let $f_{i} \in C(K, X), i=0,1,2$, be continuous functions and $\varepsilon>0$. Then there exists $\eta>0$ such that, if $x \in B\left(f_{0}(k) ; \eta\right), k \in K$, we have that $\left[B\left(f_{1}(k) ; \varepsilon / 2\right) \times B\left(f_{2}(k) ; \varepsilon / 2\right)\right] \cap \phi(x) \neq \varnothing$ (the proof is analogous to the part (2) of Lemma 6.2). Take $g \in B\left(f_{0} ; \eta\right)$ in $C(K, X)$ and define $\psi: K \rightarrow 2^{X \times X}$ as $\psi(k)=[\phi \circ g(k)] \cap\left[B\left(f_{1}(k) ; \varepsilon\right) \times B\left(f_{2}(k) ; \varepsilon\right)\right], k \in K$. As in Proposition 4.2 , it is proved that $\psi$ is lower semicontinuous and that $\psi(k)$ is a nonempty closed convex set for each $k \in K$. Applying Michael's theorem [13, p. 5], there exists a continuous selection $s=\left(s_{1}, s_{2}\right): K \rightarrow X \times X$ such that $s(k) \in \psi(k)$ for each $k \in K$. Now take $g_{i}=s_{i}, i=1,2$.

6.7. Proposition. Let $X$ be a Banach space with $B_{X}$ stable, $K$ a compact Hausdorff space and $\mu$ a positive Radon measure on $K$. Then $B_{L_{1}(\mu, X)}$ is stable. 
Proof. Take $f_{i} \in S_{L_{1}(\mu, X)}, i=0,1,2$, satisfying $f_{0}=\frac{1}{2}\left(f_{1}+f_{2}\right)$, that implies $\left\|f_{0}(k)\right\|=\frac{1}{2}\left(\left\|f_{1}(k)\right\|+\left\|f_{2}(k)\right\|\right)$ a.e. Given $0<\varepsilon \leq 1$, let us prove that there exists $\rho>0$ such that $V\left(f_{0} ; \rho\right) \subseteq D\left(f_{1}, f_{2} ; \varepsilon\right)$ in $B_{L_{1}(\mu, X)}$. If

$$
\int_{K}\left|\left\|f_{i}(k)\right\|-\left\|f_{0}(k)\right\|\right| d \mu=0, \quad i=1,2,
$$

(what implies that $\left.\left\|f_{0}(k)\right\|=\left\|f_{i}(k)\right\|, i=1,2\right)$ the conclusion follows from the proof of Proposition 6.3. Assume that $\int_{K}\left|\left\|f_{i}(k)\right\|-\left\|f_{0}(k)\right\|\right| d \mu>0$ and denote

$$
U_{i}=\left\{k \in K:\left\|f_{i}(k)\right\|>\left\|f_{0}(k)\right\|\right\}, \quad i=1,2 .
$$

Then

$$
\begin{gathered}
\int_{U_{1}}\left(\left\|f_{1}(k)\right\|-\left\|f_{0}(k)\right\|\right) d \mu=\int_{U_{2}}\left(\left\|f_{2}(k)\right\|-\left\|f_{0}(k)\right\|\right) d \mu \\
=\frac{1}{2} \int_{K}\left|\left\|f_{i}(k)\right\|-\left\|f_{0}(k)\right\|\right| d \mu=H^{\prime}>0 .
\end{gathered}
$$

Let $H=\min \left\{1, H^{\prime}\right\}$ and, as in Proposition 6.3, choose a compact $K_{0} \subseteq K$ satisfying that $f_{i}$ is continuous on $K_{0}$ and $\int_{K \backslash K_{0}}\left\|f_{i}-f_{0}\right\| d \mu \leq \varepsilon H / 32, i=$ $0,1,2$. Let $\delta=\varepsilon H / 16 \mu\left(K_{0}\right)$. By Lemma 6.6 , there exists $0<\eta<1$ such that, if $h \in C\left(K_{0}, X\right)$ and $\left\|h-f_{0}\right\|_{K_{0}} \leq \eta$, there exist $h_{i} \in C\left(K_{0}, X\right)$ satisfying

$$
\left\|h_{i}-f_{i}\right\|_{K_{0}} \leq \delta, \quad h=\frac{1}{2}\left(h_{1}+h_{2}\right)
$$

and

$$
\|h(k)\|=\frac{1}{2}\left(\left\|h_{1}(k)\right\|+\left\|h_{2}(k)\right\|\right), \quad k \in K_{0} .
$$

Let $M=\max \left\{1,\left\|f_{i}(k)\right\|_{K_{0}}: i=0,1,2\right\}$ and $\rho=H \varepsilon \eta /(32 \cdot M)$. Pick $g_{0} \in$ $V\left(f_{0} ; \rho\right)$ in $B_{L_{1}(\mu, X)}$ and put $A=\left\{k \in K_{0}:\left\|f_{0}(k)-g_{0}(k)\right\|>\eta\right\}$. Then

$$
\eta \cdot \mu(A) \leq \int_{A}\left\|f_{0}(k)-g_{0}(k)\right\| d \mu \leq \rho
$$

that is, $\mu(A) \leq H \varepsilon /(32 \cdot M)$. So we can choose a compact $K_{1} \subseteq K_{0} \backslash A$ such that $g_{0}$ is continuous on $K_{1}$ and

$$
\int_{K_{0} \backslash K_{1}}\left\|f_{i}-f_{0}\right\| d \mu \leq \varepsilon H / 8, \quad i=1,2 .
$$

Denote $U_{i 1}=U_{i} \cap K_{1}, i=1,2$, and observe that

$$
\int_{U_{i} \backslash K_{1}}\left(\left\|f_{i}(k)\right\|-\left\|f_{0}(k)\right\|\right) d \mu \leq \int_{K \backslash K_{1}}\left\|f_{i}-f_{0}\right\| d \mu \leq \frac{\varepsilon H}{32}+\frac{\varepsilon H}{8} .
$$

Thus

$$
\int_{U_{i 1}}\left(\left\|f_{i}(k)\right\|-\left\|f_{0}(k)\right\|\right) d \mu \geq H-\frac{\varepsilon H}{32}-\frac{\varepsilon H}{8} \geq \frac{27}{32} H .
$$

Let $\hat{g}_{0}: K_{0} \rightarrow X$ a continuous extension of $g_{0 \mid K_{1}}$ to $K_{0}$ satisfying $\left\|f_{0}-\hat{g}_{0}\right\|_{K_{0}} \leq$ $\eta$. By Lemma 6.6 there exist continuous functions $\hat{g}_{i}: K_{0} \rightarrow X, i=1,2$, such that

$$
\begin{aligned}
& k \in K_{0}, \quad\left\|\hat{g}_{i}(k)-f_{i}(k)\right\| \leq \delta, \quad i=1,2, \\
& \hat{g}_{0}(k)=\frac{1}{2}\left(\hat{g}_{1}(k)+\hat{g}_{2}(k)\right) \text {, } \\
& \left\|\hat{g}_{0}(k)\right\|=\frac{1}{2}\left(\left\|\hat{g}_{1}(k)\right\|+\left\|\hat{g}_{2}(k)\right\|\right) \text {. }
\end{aligned}
$$


Let $b_{i}=\int_{K_{1}}\left(\left\|\hat{g}_{i}(k)\right\|-\left\|g_{0}(k)\right\|\right) d \mu, i=1,2$, and note that $b_{1}+b_{2}=0$ and

$$
\begin{aligned}
\left|b_{i}\right|= & \left|\int_{K_{1}}\left(\left\|\hat{g}_{i}(k)\right\|-\left\|g_{0}(k)\right\|\right) d \mu\right| \\
\leq & \left|\int_{K_{1}}\left(\left\|\hat{g}_{i}(k)\right\|-\left\|f_{i}(k)\right\|\right) d \mu\right| \\
& +\left|\int_{K_{1}}\left(\left\|f_{i}(k)\right\|-\left\|f_{0}(k)\right\|\right) d \mu\right|+\left|\int_{K_{1}}\left(\left\|f_{0}(k)\right\|-\left\|g_{0}(k)\right\|\right) d \mu\right| \\
\leq & \int_{K_{1}}\left\|\hat{g}_{i}(k)-f_{i}(k)\right\| d \mu+\int_{K \backslash K_{1}}\left\|f_{i}(k)-f_{0}(k)\right\| d \mu \\
& +\int_{K_{1}}\left\|f_{0}(k)-g_{0}(k)\right\| d \mu \\
\leq & \delta \cdot \mu\left(K_{1}\right)+\varepsilon H / 32+\varepsilon H / 8+(\varepsilon \eta H) /(32 M) \leq \varepsilon H / 4 .
\end{aligned}
$$

Here we use (1), that

$$
\int_{K}\left[\left\|f_{i}\right\|-\left\|f_{0}\right\|\right] d \mu=0 \quad\left(\text { thus }\left|\int_{K_{1}}\left[\left\|f_{i}\right\|-\left\|f_{0}\right\|\right] d \mu\right|=\left|\int_{K \backslash K_{1}}\left[\left\|f_{i}\right\|-\left\|f_{0}\right\|\right] d \mu\right|\right)
$$

and that $g_{0} \in V\left(f_{0} ; \rho\right)$. Next define measurable functions $g_{i}^{\prime}: K_{1} \rightarrow X, i=$ 1,2 , satisfying

$$
\begin{array}{cc}
k \in K_{1}, \quad & g_{0}(k)=\frac{1}{2}\left(g_{1}^{\prime}(k)+g_{2}^{\prime}(k)\right), \quad\left\|g_{0}(k)\right\|=\frac{1}{2}\left(\left\|g_{1}^{\prime}(k)\right\|+\left\|g_{2}^{\prime}(k)\right\|\right) \\
\int_{K_{1}}\left\|g_{i}^{\prime}(k)\right\| d \mu=\int_{K_{1}}\left\|g_{0}(k)\right\| d \mu, \quad i=1,2 .
\end{array}
$$

If $b_{1}=b_{2}=0$, take $g_{i}^{\prime}=\hat{g}_{i}, i=1,2$. Suppose, for instance, that $b_{1}>0$ (the case $b_{2}>0$ is analogous) and denote $B=\left\{k \in K_{1}:\left\|\hat{g}_{1}(k)\right\|>\left\|g_{0}(k)\right\|\right\}$. Then $\int_{B}\left\|\hat{g}_{1}(k)\right\| d \mu \geq \int_{B}\left\|g_{0}(k)\right\| d \mu+b_{1}$. For $0 \leq \lambda \leq 1$, let $g_{\lambda}(k)=\lambda \hat{g}_{1}(k)+$ $(1-\lambda) g_{0}(k)$, that satisfies $\left\|g_{\lambda}(k)\right\|=\lambda\left\|\hat{g}_{1}(k)\right\|+(1-\lambda)\left\|g_{0}(k)\right\|, k \in K_{1}$. Thus there exists $0 \leq \lambda_{0} \leq 1$ such that $\int_{B}\left\|g_{\lambda_{0}}(k)\right\| d \mu=\int_{B}\left\|\hat{g}_{1}(k)\right\| d \mu-b_{1}$. Define $g_{i}^{\prime}, i=1,2$, by

$$
k \in K_{1}, \quad g_{1}^{\prime}(k)=\left\{\begin{array}{ll}
g_{\lambda_{0}}(k), & k \in B, \\
\hat{g}_{1}(k), & k \in K_{1} \backslash B,
\end{array} \quad g_{2}^{\prime}(k)=2 g_{0}(k)-g_{1}^{\prime}(k) .\right.
$$

\section{Note that}

(1) $g_{0}(k)=\frac{1}{2}\left(g_{1}^{\prime}(k)+g_{2}^{\prime}(k)\right),\left\|g_{0}(k)\right\|=\frac{1}{2}\left(\left\|g_{1}^{\prime}(k)\right\|+\left\|g_{2}^{\prime}(k)\right\|\right), k \in K_{1}$.

(2) $\int_{K_{1}}\left\|g_{i}^{\prime}(k)\right\| d \mu=\int_{K_{1}}\left\|g_{0}(k)\right\| d \mu, i=1,2$.

(3) We claim that $\int_{K_{1}}\left\|g_{i}^{\prime}(k)-f_{i}(k)\right\| d \mu \leq 3 \varepsilon / 4, i=1$, 2. Let us see that 
$1-\lambda_{0} \leq \varepsilon / 3$

$$
\begin{aligned}
\frac{\varepsilon H}{4} & \geq b_{1}=\left(1-\lambda_{0}\right) \int_{B}\left(\left\|\hat{g}_{1}(k)\right\|-\left\|g_{0}(k)\right\|\right) d \mu \\
& \geq\left(1-\lambda_{0}\right) \int_{U_{11}}\left(\left\|\hat{g}_{1}(k)\right\|-\left\|g_{0}(k)\right\|\right) d \mu \\
& =\left(1-\lambda_{0}\right)\left[\int_{U_{11}}\left(\left\|\hat{g}_{1}(k)\right\|-\left\|f_{1}(k)\right\|\right) d \mu+\int_{U_{11}}\left(\left\|f_{1}(k)\right\|-\left\|f_{0}(k)\right\|\right) d \mu\right. \\
& \left.\left.\geq\left(1-\lambda_{0}\right)\left(-\delta \cdot \mu\left(U_{11}\right)+\frac{27}{32} \cdot H-\rho\right)+f_{U_{11}}(k)\|-\| g_{0}(k) \|\right) d \mu\right] \\
& \geq\left(1-\lambda_{0}\right)\left(\frac{27}{32} H-\frac{\varepsilon H}{16}-\frac{\varepsilon \eta H}{32 M}\right) \\
& \geq\left(1-\lambda_{0}\right)\left(\frac{27}{32}-\frac{1}{16}-\frac{1}{32}\right) H=\left(1-\lambda_{0}\right) \frac{24}{32} H .
\end{aligned}
$$

So $\left(1-\lambda_{0}\right) \leq \varepsilon / 3$. Also, as $\left\|\hat{g}_{1}(k)-f_{i}(k)\right\| \leq \delta, k \in K_{1}$, and $\left\|f_{i}\right\|_{1}=1 \geq\left\|g_{0}\right\|_{1}$, we obtain

$$
\begin{aligned}
\int_{B}\left\|\hat{g}_{i}(k)-g_{0}(k)\right\| d \mu \\
\quad \leq \int_{B}\left\|g_{0}(k)\right\| d \mu+\int_{B}\left\|f_{i}(k)\right\| d \mu+\delta \cdot \mu(B) \leq 2+\frac{\varepsilon H}{16}
\end{aligned}
$$

and therefore

$$
\begin{aligned}
& \int_{K_{1}}\left\|f_{i}(k)-g_{i}^{\prime}(k)\right\| d \mu \leq \int_{K_{1}}\left\|f_{i}(k)-\hat{g}_{i}(k)\right\| d \mu \\
& \quad+\int_{B}\left\|\hat{g}_{i}(k)-\left(\lambda_{0} \hat{g}_{i}(k)+\left(1-\lambda_{0}\right) g_{0}(k)\right)\right\| d \mu \\
& \leq \delta \cdot \mu\left(K_{1}\right)+\left(1-\lambda_{0}\right) \int_{B}\left\|\hat{g}_{i}(k)-g_{0}(k)\right\| d \mu \leq \frac{\varepsilon H}{16}+\frac{\varepsilon}{3}\left(2+\frac{\varepsilon H}{16}\right) \leq 3 \varepsilon / 4 .
\end{aligned}
$$

Finally define $g_{i}, i=1,2$,

$$
k \in K, \quad g_{i}(k)= \begin{cases}g_{i}^{\prime}(k), & k \in K_{1}, \\ g_{0}(k), & k \in K \backslash K_{1} .\end{cases}
$$

\section{Then we have}

(a) $k \in K, g_{0}(k)=\frac{1}{2}\left(g_{1}(k)+g_{2}(k)\right)$ and $\left\|g_{0}(k)\right\|=\frac{1}{2}\left(\left\|g_{1}(k)\right\|+\left\|g_{2}(k)\right\|\right)$.

(b) $\int_{K}\left\|g_{i}(k)\right\| d \mu=\int_{K}\left\|g_{0}(k)\right\| d \mu \leq 1, i=1,2$. 
(c) For $i=1,2$, we have

$$
\begin{aligned}
& \int_{K}\left\|f_{i}(k)-g_{i}(k)\right\| d \mu \\
&=\int_{K \backslash K_{1}}\left\|f_{i}(k)-g_{0}(k)\right\| d \mu+\int_{K_{1}}\left\|f_{i}(k)-g_{i}(k)\right\| d \mu \\
& \leq \int_{K \backslash K_{1}}\left\|f_{i}(k)-f_{0}(k)\right\| d \mu+\int_{K \backslash K_{1}}\left\|f_{0}(k)-g_{0}(k)\right\| d \mu \\
&+\int_{K_{1}}\left\|f_{i}(k)-g_{i}(k)\right\| d \mu \\
& \leq \frac{\varepsilon H}{32}+\frac{\varepsilon H}{8}+\rho+\frac{3 \varepsilon}{4}<\varepsilon .
\end{aligned}
$$

Thus $V\left(f_{0} ; \rho\right) \subseteq D\left(f_{1}, f_{2} ; \varepsilon\right)$.

6.8. Proposition. Let $X$ be a Banach space with $B_{X}$ stable and $(\Omega, \Sigma, \mu)$ a positive measure space. Then $B_{L_{1}(\mu, X)}$ is stable.

Proof. Use the Stone compact corresponding to the measure algebra $(\Sigma, \mu)$ as in Proposition 6.4.

6.9. Proposition. Let $\left\{X_{i}\right\}_{i \in I}$ be a family of Banach spaces with $B_{X_{i}}$ stable. Then, if $Y=\left(\sum_{i \in I} \bigoplus X_{i}\right)_{1}, B_{Y}$ is stable.

Proof. The proof, analogous to that of Proposition 6.5 (with some changes, as in Proposition 6.7), is left to the reader.

Open problem. Suppose that $B_{X}$ is US. Is $B_{L_{p}(\mu, X)}$ US, $1 \leq p<\infty$ ?

\section{REFERENCES}

1. E. M. Alfsen, Compact convex sets and boundary integrals, Springer-Verlag, 1971.

2. R. M. Blumenthal, J. Lindenstrauss and R. R. Phelps, Extreme operators into $C(K)$, Pacific J. Math. 15 (1965), 747-756.

3. A. Clausing, Retractions and open mappings between convex sets, Math. Z. 160 (1978), 263-274.

4. A. Clausing and S. Papadopoulou, Stable convex sets and extremal operators, Math. Ann. 231 (1978), 193-203.

5. R. Grzaslewicz, Finite dimensional Orlicz spaces, Bull. Polish Acad. Sci. Math. 33 (1985), 277-283.

6. E. Lacey, The isometry theory of classical Banach spaces, Springer-Verlag, 1974.

7. A. Lazar, Spaces of affine continuous functions on simplexes, Trans. Amer. Math. Soc. 134 (1968), 503-525.

8. A. Lima, Intersection properties of balls and subspaces in Banach spaces, Trans. Amer. Math. Soc. 227 (1977), 1-62.

9. J. Lindenstrauss, Extension of compact operators, Mem. Amer. Math. Soc., no. 48, 1963.

10. J. Lindenstrauss and L. Tzafriri, Classical Banach spaces, Lecture Notes in Math., vol. 338, Springer-Verlag, 1973.

11. P. D. Morris and R. R. Phelps, Theorems of Krein-Millman type for certain convex sets of operators, Trans. Amer. Math. Soc. 150 (1970), 183-200.

12. S. Papadopoulou, On the geometry of stable compact convex sets, Math. Ann. 229 (1977), 193-200. 
13. T. Parthasarathy, Selection theorems and their applications, Lecture Notes in Math., vol. 263, Springer-Verlag, 1972.

14. D. Sentilles, Decomposition of weakly measurable functions, Indiana Univ. Math. J. 32 (1983), 425-437.

15. M. Sharir, Characterization and properties of extreme operators into $C(Y)$, Israel J. Math. 12 (1972), 174-183.

16. D. Werner, Extreme points in function spaces, Proc. Amer. Math. Soc. 89 (1983), 598-600.

Facultad de Matemáticas, Departamento de Análisis Matemático, Universidad ComPLUTENSE DE MADRID, 28040-MADRID, SPAIN 